Cinémas

Revue d'études cinématographiques

Journal of Film Studies

\title{
Corps filmique entre lisible et visible chez Marguerite Duras
}

\section{Marie-Françoise Grange}

Volume 3, numéro 1, automne 1992

URI : https://id.erudit.org/iderudit/1001183ar

DOI : https://doi.org/10.7202/1001183ar

Aller au sommaire du numéro

Éditeur(s)

Cinémas

ISSN

1181-6945 (imprimé)

1705-6500 (numérique)

Découvrir la revue

Citer cet article

Grange, M.-F. (1992). Corps filmique entre lisible et visible chez Marguerite Duras. Cinémas, 3(1), 115-124. https://doi.org/10.7202/1001183ar

\section{Résumé de l'article}

L'auteure aborde le film Césarée de Marguerite Duras (1979), comme lieu privilégié de l'émergence du travail de l'écriture filmique. En refusant de résoudre l'écartement, les ruptures, le film table sur le conflit et plutôt que de le taire, le fait proliférer. Le lisible devient comme autre du visible et le visible, autre du lisible. 


\title{
Corps filmique entre lisible et visible chez Marguerite Duras
}

\section{Marie-Françoise Grange}

\begin{abstract}
RÉSUMÉ
L'auteure aborde le film Césarée de Marguerite Duras (1979), comme lieu privilégié de l'émergence du travail de l'écriture filmique. En refusant de résoudre l'écartement, les ruptures, le film table sur le conflit et plutôt que de le taire, le fait proliférer. Le lisible devient comme autre du visible et le visible, autre du lisible.
\end{abstract}

\begin{abstract}
The author approaches the film Césarée by Marguerite Duras, as a privileged position from which to stydy the emergence of a work of film writing. Refusing to resolve the distance and the ruptures, the film is based on conflict and rather than hide them, the film chooses to multiply them. Thus, that which is written becomes other than that which is seen, and that which is seen, becomes other thanthat which is written.
\end{abstract}

Le film, qu'il soit signé par Marguerite Duras ou tout autre réalisateur, semble toujours écartelé entre ses différentes pistes signifiantes. À la fois image, texte verbal, bruit, musique, texte écrit, il se façonne dans cette multiplicité intrinsèque. Le cinéma, écrit Marie-Claire Ropars, "se définit par la mise en montage d'un dispositif pluriel» (1983: p. 71). Le film jouera de cette pluralité pour construire un texte multidirectionnel que certains auteurs, telle Marguerite Duras, auront plaisir à exploiter, à travailler.

Césarée de Marguerite Duras, court métrage réalisé en 1979 et texte littéraire publié la même année, constitue un double texte dont l'un, le film, rejoue en lui-même cette double appartenance. 
Le texte littéraire n'y est-il pas devenu partie intégrante de la bande-son?

La bande image du film présente une succession de plans consacrés pour la plupart à des statues, situées sur l'Esplanade du Louvre à Paris, dont certaines sont de Maillol. La bande son est un texte autour de Bérénice écrit, lu par Marguerite Duras, ponctué par un violon. L'absence de son synchrone marque le partage entre son et image. Dans la structure plurielle du film s'insinuent ainsi d'autres découpes, ne se reliant pas nécessairement, terme à terme : du son à l'image, des mots aux choses, du dire au voir, du lire à l'écrire, du lisible au visible. Multiples découpes incitant des passages, des traversées productrices de sens ou de non-sens, frayant un trajet entre différents textes constitutifs du texte filmique.

Tout commence bien évidemment, dans Césarée, par cette rupture flagrante entre une bande-son et une bande-image. Flagrante, car cette rupture saute au yeux tout comme elle s'entend. Elle s'affiche en plaquant plusieurs espaces hétérogènes, celui de l'énonciation verbale d'où le texte littéraire se profère, et celui d'une représentation hachée par de faux raccords. La voix off (et non hors champ), par définition sans corps, est exclue de l'espace désigné par l'image. Elle flotte au-dessus d'un voir qui ne renvoie pas au lieu d'où elle parle. L'écart est creusé par l'absence, celle de son synchrone, mais également celle de corps récitant. Pas d'appartenance à une même fiction : jamais la circulation à l'intérieur de l'espace montré ne sera attribuable au regard du personnage lisant. Pas de promeneur donc lisant un livre et visitant, en même temps, les jardins du Louvre. Le lieu de production du son s'affirme comme irrémédiablement différent du lieu de tournage.

Radicale exclusion, la voix s'énonce en intérieur, les plans sont d'extérieur. Il y aurait une «coupure» entre les signifiants émis sur chacune des deux pistes. En tout cas il y aurait volonté d'intégrer, à l'intérieur du film, la marque d'une rupture ou plutôt d'un espacement susceptible de laisser jouer les éléments les uns vis-à-vis des autres. Sans trop vouloir insister sur la cassure - ce qui risquerait de nous faire rater l'enjeu des relations différentielles - il me semble important de souligner, d'une part, l'inscription de l'écart, d'autre part, le fait que cette inscription passe par le refus de donner en pâture au spectateur des signifiants visuels ou sonores aptes à faire fonctionner bande-son et bandeimage dans une temporalité et un espace diégétiques communs.

Le son d'un côté, l'image de l'autre, la voix d'un côté, la vue de l'autre : double partage. Mais le son est aussi musique comme le texte est interprétation vocale, les deux composantes sonores (voix 
et violon) se redoublent, se cherchent et s'opposent, se retrouvent et s'unissent dans la partition musicale qui se jouerait à deux voix. Parler de bande-son empêche alors d'éliminer l'autre de la voix. Certes, il y a celle de Marguerite Duras, ce qui n'est pas sans incidence par ailleurs, et il y a celle du violon, contrepoint, aspirant le vocal dans le musical. Il n'y a pas sur le son, il n'y a pas dans la voix, que de la voix et l'autre du son fait poindre l'autre de la voix. Double parole et cela à plus d'un titre.

La voix dit le texte écrit, les mots. Elle est formulation et opère pour cela une transformation Elle réalise une interprétation ce qui, inévitablement, implique une transcription. Car la voix est résonance, elle livre le corps sans le montrer, elle délivre sa chair, son passé. «Ce passage par la voix (...) produit les effets de sens dont toute une lecture atteste dès lors qu'elle est re-lecture et déchiffrement d'un texte» (Vasse, p. 218). La phrase prononcée se double d'un autre énoncé, la double parole est double texte. Le lisible des mots du texte littéraire sollicitant une lecture se fait déborder par le corps insistant de son interprète. La «vocalisation» fait glisser l'ensemble vers quelque chose de différent, ou plutôt il serait plus juste de dire fait glisser l'ensemble vers ce qui, en lui, est différent. Nous assisterions à une opération de dévoilement. Par la lecture, l'insertion d'un corps étranger, l'écriture offre ce qui, sournoisement, la travaille. Sans lecture, pas d'écriture; sans interprétation, pas de sens; sans vibration, pas d'indécidabilité, pas de «reste». Si la voix me semble relever de cette dimension du «reste», à elle donc de porter, de redonner la fêlure du texte. Le lisible, ce qui est à lire, se fait doubler par sa lecture qui le déplace de sa plénitude illusoire. Le lisible, à lire, exposer dans son processus de lecture, se décale. Le lu, à son tour, ne recouvre pas ce qui le suscite, mais fait éclore, à l'intérieur de l'écrit, les tensions, les forces en présence qui le poussent, en dehors de son unité, en dehors de lui-même. Le lu comme l'autre du lisible, le reste du mot, de l'énoncé, le surplus, ne devrais-je pas dire le supplément?

Car l'on sait que «[le supplément] vient toujours en excès pour une défaillance, suscitant sans repos, selon un mouvement oblique et fatal, des effets de manque et de désirs de présence pleine» (Lévesque, p. 132), aussi «l'écriture inlassablement appelle et commande un supplément de lecture qui appelle et commande un supplément d'écriture» (p. 133). La lecture écrit le texte, le réécrit dans ce mouvement de différence restituant à l'écriture sa fracture interne. Répondrait à ce lisible, désigné comme tel (cf. le débit de la voix, ainsi que la littérarité de la parole entendue), une lecture (désormais lecture-écriture) délivrant le corps textuel de l'illusion de sa plénitude, de sa suffisance. Émergerait alors une 
zone «d'oscillation indécidable» selon l'expression de Sarah Kofmann (p. 99). La lecture, en exposant le lisible du texte littéraire, le crève et laisse surgir l'espacement dans lequel se noient les mots. Il y a du lisible mais le lire l'entraîne, par une étrange dérive, vers sa propre césure qu'il faudrait rapprocher de celle du visible, entre visible et visuel, que Georges DidiHuberman a remarquablement su mettre en valeur dans son livre Devant l'image. Cette déchirure, c'est ni plus ni moins ce que nous offre le travail du film, capable de monter, de faire entendre et de montrer la double scène où ce qui se donne à la lecture voit, par la lecture, ses certitudes se déconstruire.

Le travail du film nous offrirait ce double mouvement. Sur le son, dans un premier temps : unifier, reconstruire, transmettre tel quel un texte littéraire sans altération, ce qui revient à dire sans altérité. Que la voix soit celle de Duras, au timbre fort reconnaissable, relève probablement de ce désir de réduction de l'écart, de suppression de l'autre dans le même. Sorte de garantie, pacte de non-trahison, la lecture par l'auteure de son propre texte littéraire éliminerait tout risque d'agression de l'extérieur. Poser une mainmise en contresignant, par la voix, le texte serait une façon de diminuer les dégâts, de minimiser la fêlure. Mais le film en dévoilant la scène de lecture libère l'écriture et lui confère des accents plus derridiens. Autre mouvement : la parole se scinde, le texte se dédouble et l'écriture est lecture, la lecture est écriture. La logique du supplément «veut que le dehors soit dedans, que l'autre et le manque viennent s'ajouter comme un plus qui remplace un moins, que ce qui s'ajoute à quelque chose tienne lieu du défaut de cette chose» (Derrida, p. 308). Déjà sur le son se trace, dans le film, cette double bande que le rapport son/image va porter, déporter.

Le off semble tout particulièrement se glisser dans cette marge. À la fois présent et absent, le son désigné ainsi par son rapport à l'image et non par une caractéristique qui lui serait propre s'offre comme dehors et dedans. Sa simultanéité le fait rôder sur et autour. Non localisable et pourtant là, ici et ailleurs, le off occupe une étrange position. Lié à l'image, si ce n'est à un premier niveau par son nom, il en porte la marque de l'exclusion. "Mélange de vacuité et de densité, de maîtrise et de malaise» (Ropars, 1990 : p. 111), ballottée, la parole, énoncée dans cette dépossession d'ellemême, se donne comme symptôme d'une cassure, d'un éraillement. Nous pourrions, à ce moment-là de l'analyse, reprendre le point de vue de Marie-Claire Ropars sur la capacité du cinéma de réfléchir «la désunion du langage» (p. 111). Je ne crois pas utile de paraphraser une démonstration aussi brillante, me contentant tout simplement d'y référer. En revanche, il me semble intéressant 
d'examiner comment, dans ce film de Marguerite Duras, s'imbriquent des strates dont l'empilement, la jonction/disjonction, la synthèse disjonctive diraient Deleuze et Guattari, conduisent à l'émergence d'une défaillance de l'option totalitaire de la machine textuelle. Comment l'autre du lisible et l'autre du visible se recouvrent pour mieux se découvrir comme non compressibles l'un par l'autre? Et si nous avons vu comment la voix de l'auteureréalisatrice déraille, portant écho d'une brisure interne, reste à montrer comment le fonctionnement du système sonore se retrouve, mutatis mutandis, dans le fonctionnement de l'image, afin d'étendre ensuite la démonstration à l'ensemble du texte filmique.

La lecture, en effet, est multiple. Tout d'abord, d'un point de vue général, comme je le faisais remarquer un peu plus haut, elle est interprétation, elle s'ouvre ainsi vers tous les possibles interprétatifs. C'est un fait de lecture qu'il y en a toujours plusieurs. Ensuite, si elle est du côté du lisible, du texte organisé à partir d'énoncés linguistiques, elle est également du côté du visible, c'est-à-dire du côté de l'image, de la représentation.

De la description rapide faite auparavant de la bande-image de Césarée a été retenu, comme élément principal, le parcours que l'image cinématographique propose à travers un certain nombre de statues. Ce trajet est effectué par une suite de travellings et par le biais du montage, rapprochant/écartant ces statues les unes des autres. Il y a d'une part circulation à travers un espace référentiel (l'esplanade du Louvre, place de la Concorde et quai de Seine), circulation construisant un espace diégétique, celui du film, et d'autre part le fait que cette circulation opère des liens, incite des passages entre images, entre représentations d'un plan à l'autre. Ce qui revient à dire que là encore se développe un processus de lecture, mais cette fois-ci de sculptures.

$\mathrm{Si}$ la sculpture en tant «qu'élément de représentation» (DidiHuberman, p. 26) opère dans la dimension du visible, l'image filmique, ayant sa propre organisation interne, travaille dans la même sphère. Et nous voici confrontés à deux visibles, celui de l'objet filmé, déjà représentation (sculpture), et celui de la vue filmique, image de cette représentation sculpturale. Deux visibles dont l'un, celui du film, n'aurait pour prétention que de présenter l'autre et du même coup disparaîtrait derrière l'autre. Présentation de représentation, simple mise en espace, mise en scène de représentation, le film ne ferait alors que montrer le visible de la sculpture, le rendre visible. En l'offrant aux yeux du spectateur, il s'accorderait le rôle de révélateur. Pas de commentaire fallacieux mais un dévoilement aussi impartial et neutre qui soit. Si la voix de l'auteure garantissait la vérité et l'authenticité de la lecture du texte littéraire, l'analogie de l'image cinématographique, en 
effaçant le visible filmique, se porterait garante de l'absence d'interprétation. Sans vouloir reprendre le débat sur la transparence de l'image, ni refaire les démonstrations effectuées en leur temps, c'est une évidence que l'image cinématographique ne transmet pas, brut, le réel filmé. Et le spectateur, comme le souligne Marc Vernet (p. 5), est conscient de cette opacité de l'image dont il tire plaisir. Corrélativement, l'impression de réalité fonctionne. L'image de sculpture est, certes, une image mais une bonne image qu'il plaît au spectateur de voir et de ne pas voir en tant que telle. Cette double croyance tire le spectacle dans plusieurs sens.

Il y aurait va-et-vient entre, d'une part, la négation du travail spécifique du film qui déplace une organisation sculpturale, la transforme et la fait apparaître sous d'autres configurations (filmiques) et, d'autre part, la reconnaissance du processus de lecture qui, en transportant la forme sculpturale d'une structure à une autre, la ressaisit en d'autres liens formels. Le refus de voir l'image (transparente) serait contrebalancé par l'acceptation (opacifiante) de l'idée d'une lecture. Mais attention! là aussi il peut y avoir plusieurs types de lecture. Cette lecture serait plus facilement acceptée à condition d'être envisagée dans la fidélité de la transposition que l'image cinématographique permettrait au nom de son réalisme ontologique ${ }^{l}$. Lire le visible, par visible interposé, ne serait qu'un moyen de le visibiliser, de le faire apparaître. Et nous voilà revenus pratiquement à la case précédente. Pas d'image (filmique) ou une image qui ne serait qu'opérateur d'une autre visibilité que la sienne. C'est une façon d'envisager le problème, façon qui me semble fonctionner dans la perception du film. Mais en même temps, immédiatement, se dégage une autre dimension. Il peut plaire de ne voir dans l'image que son référent ou de n'en voir que la ressemblance avec son référent, mais quelque part l'image, comme le rappelle Marc Vernet, se montre aussi.

Elle se montre d'autant plus que l'enchaînement d'un plan à l'autre n'est pas couvert par l'argumentation diégétique. Il est un fait que Césarée ne propose pas la mise en scène, dans son image, d'événements, d'actions, de personnages, bref de tout ce qui fait ordinairement ce qu'il est coutume d'appeler une «bonne» fiction. Césarée joue plutôt la carte de la déconstruction. Vidée de tout mouvement autre que celui de la caméra, le montage de l'image n'est jamais motivé par un regard, un déplacement, un passage, un corps, par quelque chose qui aiderait à en effacer la marque. Bien au contraire, la rupture entre les plans semble parfois tout particulièrement insistante. C'est le cas, au début du film : la promenade proposée entre les sculptures de Maillol sur l'esplanade du Louvre s'enchaîne, de travelling en travelling, par 
l'intermédiaire de faux raccords. Or le faux raccord va à l'encontre des habitudes de montage du cinéma classique. En conséquence il se voit et visualise le montage comme fracture, déchirure de l'image. Le passage d'un plan à l'autre s'y affiche en tant que tel. Et surtout, le raccord non classique met en valeur cette inscription de la rupture qui se loge dans le passage d'une image à l'autre. Si l'enjeu même de tout raccord est cette liaison dans la déliaison, il demeure possible d'insister sur, ou d'effacer cette petite dissonance qui casse une continuité diégétique, une continuité de représentation, qui contrarie l'idée même de passage et de constitution d'une totalité unitaire. Le visible du film ne s'efface plus derrière le visible de la sculpture, même si les deux sont parfaitement imbriqués l'un dans l'autre. Il fait écran. Il affiche la fracture dans laquelle il redécoupe le visible de la sculpture, l'obligeant à se déployer de-ci, de-là, dans le plan ainsi qu'entre les plans. Il le casse et inévitablement le rate. Il le livre à d'autres rythmes, le refond, le fait glisser le long de nouvelles chaînes signifiantes. Il lui impose d'autres liens, d'autres frayages, il le forme en le déformant. Que ce soit par insertion dans un autre espace, par inscription dans la durée du plan ou par redécoupages incessants dus au point de vue multiple et variable de la caméra, il démembre la sculpture en la prenant dans d'autres configurations. Le visible filmique lit et en lisant réécrit le visible sculptural. Que la lecture soit loin d'être innocente est un fait, mais elle a principalement pour effet de libérer, par l'écriture filmique, par le nouveau jeu formel qu'elle propose, des forces internes qui refusent de rentrer dans l'économie d'une simple restructuration. Et s'il y a toujours un reste qui refuse la transcription, s'il existe toujours un autre qui refuse les puissances nivelantes du même, la lecture-écriture saisit et dessaisit la mouvance figurale du sculptural par et dans la mouvance figurale du filmique. Comme si des espaces d'indécidabilité se devaient d'être ménagés, des espaces de visibilité non encore pétrifiée, de figurabilité dans lesquels un ensemble se retire de sa totalité unifiée. Le visible du film impulse à la sculpture ce mouvement de retrait, il la fait disparaître et transparaître dans une image qui n'est plus son image. Le visible du film est le visible de l'autre et impose à l'autre d'être le sien. Nous pénétrons, immanquablement, par une telle imbrication, dans une logique de trace scripturale où l'un, en portant l'autre, devient son autre. Le visible du film transporte sa fracture interne. Visible de visible devenu trace de visible, il est exproprié de son unité. Sa constitution en strates qui le fait, ici, représentation de représentation, lui fait composer l'autre en s'insinuant dans l'autre. Ce mouvement entraîne le film dans un processus où montrer un visible revient à élaborer son 
propre visible dans celui de l'autre, à diffracter une organisation dans une autre et à construire son organisation et celle de l'autre dans la diffraction. Ce qui revient à dire que le film déploie sa fracture à plusieurs niveaux. Il est écart, espacement dans cet effet de vis-à-vis qui dédouble son visible, dans cette problématique de trace dans laquelle il est pris et, plus fondamentalement, il est écart, espacement dans sa structure langagière même.

Par le montage, le film est point de vue multiple et construit sa représentation (de représentation) à travers ces césures internes qui insufflent, dans la jonction, le conflit des vues. Pas de montage additionnel mais conflictuel comme l'affirme Marie-Claire Ropars, commentant Eisenstein. Penser en terme de conflit et non d'addition le lien entre les différents constituants du film suppose d'évincer l'idée de totalité et d'introduire la notion d'un "processus discontinu et différentiel» (Ropars, 1981 : p. 43) sous la représentation, sous le visible et, pour être fidèle au discours roparsien, sous le signe linguistique procédant à la constitution du sens, donc sous le lisible au sens strict. Le film produirait son sens et sa représentation, son lisible et son visible, par-dessus les tensions, relations, interactions, courts circuits. Mais admettre une telle proposition n'est-il pas étouffer un paradoxe? Si tensions il y a, le lisible et le visible qui reposent sur une mise en ordre, sur la stabilité d'une organisation, ne pourraient être obtenus que par suppression du morcellement, de la différence, de la trace et de la discontinuité. Ils ne pourraient opérer que par le rétablissement d'une totalité. Or le film joue la carte de la fracture, de la trace, de l'intervalle. Selon les thèses roparsiennes, le film expose le travail de l'écriture, "sa double pente ${ }^{2}$ ", son désir et sa négation de représentation et de signification. Et lisible et visible doivent se penser en même temps que la possibilité contenue ou affirmée de leur démantèlement. Pas d'organisation plaquée sur le chaos mais une dynamique qui se fait et se défait, qui se tisse le long d'enchaînements multiples, qui lie, relie et délie les fils de son tissage. Le texte durassien semble tout particulièrement exploiter cette énergétique oscillatoire qui restitue au film sa dimension scripturale.

Césarée est certainement un excellent exemple de cette émergence du travail de l'écriture filmique. En refusant de résoudre l'écartement, les ruptures, le film table sur le conflit et plutôt que de le taire le fait proliférer. Jamais dans le film le son et l'image ne nous raconteront la même histoire - sauf peut-être, au dernier plan, ce qui aura pour conséquence immédiate d'arrêter le film - et pourtant, pris dans la simultanéité du déroulement des pistes signifiantes, toujours le dit et le voir se heurtent afin d'ouvrir des passages dans lesquels le sens circule de 
représentations à énoncés linguistiques et vice versa. La simultanéité ménage des traversées de lisibilité et de visibilité entre son et image : par le texte verbal, l'image deviendrait lisible; par l'image, le texte verbal construirait son visible. Mais la distance, l'espacement, pour reprendre la terminologie derridienne, irrémédiablement repousse, systématiquement, ces tentatives de frayages, les empêche de fonctionner tout en les sollicitant. Jamais le film ne jouera le registre de la complémentarité des signifiants visuels et sonores. Il y aura toujours un reste, un supplément, un visible qui ne rentrera pas dans le jeu des mots et refusera ainsi de se faire lire par le texte verbal, un texte verbal qui ne se stabilisera pas dans la référence que pourrait lui proposer le visible de l'image. S'il y a nomination et par contre coup, appel à ancrer cette nomination dans la représentation montrée, l'accord n'est pas réalisé. Même si, parfois, les énoncés iconiques et les énoncés linguistiques croisent quelques signifiants communs. Sans entrer dans le détail de l'analyse, je voudrais simplement, en guise de conclusion, pointer ce "processus discontinu et différentiel» évoqué plus haut.

Au plan huit, le bleu des colonnes de marbre tel que mentionné par le son rencontre, sur l'image, le bleu du ciel et de la bâche enveloppant la sculpture en restauration. Le son désigne la couleur bleue et de ce fait guide la lecture de l'image. L'image, en retour, visualise et lit le bleu du son. Le marbre, désormais, porte le bleu du ciel et/ou de la toile. De quel bleu s'agit-il ? De tous, évidemment, ce qui signifie d'aucun. Quant à l'image, elle affiche, en priorité, sa dominante bleue et si la pierre de la sculpture est grise ce que l'on voit, lit et remarque, c'est qu'elle ne soit pas de la couleur annoncée par le son. Le spectateur se retrouve devant un chassé-croisé : la pierre grise contre/pour le marbre bleu à la couleur de ciel et/ou de toile. Et le texte rebondit.

Visible et lisible se débordent et repoussent les limites de leur confluence. Terrain privilégié de l'union dissonante, le film durassien favorise le jeu des termes. Aussi, plutôt que de se fixer, le sens se trace et s'échappe dans les relations mises en jeu par la non-coïncidence des éléments. Ni le conflit ni l'entrechoquement des forces textuelles ne sont tus, alors les poussées signifiantes et le jeu figural délivrent leur puissance déflagrante.

Université Jean Monnet de Saint-Étienne 


\section{NOTES}

1 Selon l'expression d'André Bazin.

2 Selon le titre d'un des chapitres du livre de Marie-Claire Ropars, Le Texte divisé.

\section{OUVRAGES CITÉS}

Derrida, Jacques. De la grammatologie. Paris : Minuit, 1967.

Didi-Huberman, Georges. Devant l'image. Paris : Minuit, 1990.

Kofmann, Sarah. "Ça cloche». Les fins de l'homme. À partir du travail de Jacques Derrida. Paris : Galiléc, 1981.

Lévesque, Claude. L'Étrangeté du texte. Paris : UGE, 1978.

Ropars, Marie-Claire. Le texte divisé. Paris : PUF, 1981.

Ropars, Marie-Claire. «Le film lecteur du texte», Hors cadre 1 (1983).

Ropars, Marie-Claire. «Christian Metz et le mirage de l'énonciation». Iris (1990: «Christian Metz et la théorie du cinéma»). Paris : Méridiens Klincksieck, 1990.

Vasse, Denis. L'Ombilic et la voix. Paris : Seuil, 1974.

Vernet, Marc. Figures de l'absence. Paris : Cahiers du Cinéma, 1988. 